\title{
The Social Elements of Lookism Resulting to Body Deformity
}

\author{
Byung-Chul Lee ${ }^{1}$ \\ ${ }^{1}$ Professor, College of General Education, Silla University, Korea, labcla6@silla.ac.kr
}

\begin{abstract}
The modern society makes frequent exposures for regular aesthetic criterion, both consciously or unconsciously, and requires its community members to follow this trend. With a plethora of familiarly persisting aesthetic criterion and beauty, people grow to regard the aesthetic criterion as a formula and want to live by following that criterion. This process naturally encourages people to have cosmetic surgery and socially prevalent lookism leads to social ills beyond personal problems. It is true that plastic surgery market is becoming larger and expanded into various types day after day. A survey was conducted to current high school students and university students, and based on this, data presentation and analysis were led. Through the contents of the survey, the realistic image required by the society was presented. In the reality that plastic surgery is walking the path of social universalization, it is necessary to consider the factors that change the consciousness and the problem of deficiency that causes physical transformation. This approach is expected to serve as an opportunity to understand the appearanceism of today's youth, who have been exposed and aggravated as aesthetic standards in the mass media and social dimension. Considering that mass media have an effect on perceptual domain and physical images derived from each and every person's physical difference, the ripple effect must be much bigger. Consequently, mass media is required to provide the various educational values by considering the healthy physical mechanism which can be both realistic and individual. Most of all, the social perception shift is required but this shift cannot be realized without preceding each and every person's alteration of consciousness. Therefore, it is necessary to find and develop everyone's personality and beauty instead of judging the aesthetic criterion with a fixed framework.
\end{abstract}

Keywords: Plastic Surgery Culture, Alienation and Isolation, Premature Plastic Surgery, Employment Plastic Surgery, Exposure of Aesthetic Criterion, Influence of Mass Media

\section{Introduction}

In a reality where plastic surgery is deeply entrenched in society, the desire to pursue physical changes is being embraced in everyday life without much resistance[1]. Since there is a desire to be pretty, the desire to pursue physical change is not much different regardless of age. This consciousness gives growing youth a false perception of body modification.

The social spread of plastic surgery that causes body transformation can be judged as a trend in modern society. However, the tendency of plastic surgery to be lightly perceived as "the value of life, that is, competitiveness, social success, an event," has many side effects[2][3]. Moreover, the youth period, when they are sensitive to appearance and fashion, can shake their discernment, so it is easy to blindly attach meaning to or standardize the aesthetic standards exposed through the mass media.

Considering such a reality, there is clearly a problem to be solved. As a result, it is a matter of how much interest young people have in plastic surgery, and what kind of perception and content they have. Of course, on a social level, personally, the reasons vary. This study was presented based on the

Received: April 29, 2021; $1^{\text {st }}$ Review Result: June 12, 2021; $2^{\text {nd }}$ Review Result: July 31, 2021

Accepted: August 30, 2021 
contents of the questionnaire in the main body. The approach seeks to uncover it by targeting high school and college students through written surveys.

The reason why the youth choose plastic surgery for job search could be associated to their desire and conformity to the kind of aesthetic standard spread by the media[4]. There is no limit to the excessive consciousness of wanting to change your appearance while putting your body at risk. For example, plastic surgery for self-satisfaction has a risk of exposure to repeated plastic surgery addiction, and the resulting lawsuits and side effects bring conflicts in society as well. This can lead to increased social conflict costs as well as fatigue in the balanced development of a competitive society.

Even now, familiar and similar standards of appearance are presented through the media, and such people appear. In order to meet the aesthetic standards, the media also introduces special exercises and methods set according to them. Social isolation due to the idea of not being able to adapt to change. There is also the problem of being excluded from peer groups. In fact, the spread of this awareness can lead to bullying and depression due to appearance, as well as atrophy and maladaptation to social life among adolescents. Therefore, this study starts with the process of examining the social factors of lookism that cause body transformation and attempts to understand these pathological phenomena and changes in social consciousness and their causes[5][6].

Youth are still in the growth stage, so the risk of side effects is bound to be greater. Because it is a physical and mental problem, the impact is never small. Through the increase of the plastic surgery population, it is possible to understand early plastic surgery, which involves performing plastic surgery for self-satisfaction while suffering significant physical side effects, and employment molding as a means of livelihood such as employment[7]. Focusing on the results and analysis of the survey data, which is the influence of early plastic surgery and the media, changes in social awareness and factors on plastic surgery and employment plastic surgery will be presented. This approach is expected to serve as an opportunity to understand the lookism of today's youth, who have been exposed and aggravated as aesthetic standards in the mass media and social dimension.

In addition, in principle, in this paper, youth encompass young people. The Basic Juvenile Act refers to persons aged 9 to 24 years old. In the Juvenile Protection Act, it also refers to a person under the age of 19. Given this general meaning, the subjects of this study were sampled based on current high school students and university students. [Table 1] and [Table 2] provide details on the date, survey subject, method, number of questions, and question content.

[Table 1] Gyeonggi-do H High School

\begin{tabular}{|c|c|}
\hline Date & 2019.9.26. \\
\hline Subject & $\begin{array}{l}\text { Gyeonggi-do H High School } \\
271 \text { female students among 1st and 2nd graders } \\
\text { (6th class in 1st year, 3rd class in 2nd year) }\end{array}$ \\
\hline Method & Written Survey \\
\hline Number of Questions & 7 questions (multiple choice: 6 , subjective: 1 question) \\
\hline $\begin{array}{l}\text { Question } \\
\text { Contents }\end{array}$ & $\begin{array}{l}\square \text { Your thoughts on plastic surgery for cosmetic purposes } \\
\square \text { Reasons for people's plastic surgery and factors influencing their choice } \\
\square \text { Reasons for plastic surgery of youth and factors affecting their choice } \\
\square \text { Whether legal sanctions related to plastic surgery are necessary } \\
\square \text { Degree of influence of appearance on employment in Korea } \\
\square \text { Areas where job seekers think plastic surgery is necessary for employment } \\
\square \text { Whether there is a need for an evaluation method that does not involve appearance in the } \\
\text { recruitment process }\end{array}$ \\
\hline
\end{tabular}


[Table 2] Metropolitan S University

\begin{tabular}{c|l}
\hline Subject & 2019.11.12. 2018.11.14. \\
\hline Method & 128 female students from S university in metropolitan \\
\hline Number of Questions & Written Survey \\
\hline Question Contents & Multiple choice: 4 questions \\
\hline & $\square$ The aesthetic standard of the forehead in your opinion \\
& $\square$ The aesthetic standard for your eye size \\
& $\square$ The aesthetic standard of your lips \\
& $\square$ The aesthetic standards for your face shape
\end{tabular}

\section{Growth of Plastic Surgery Population and Change of Consciousness}

In these days, when the development cycle of medical technology is rapidly changing, plastic surgery is filling an area of society to satisfy the desire to become more beautiful and receive attention[8]. In particular, their age is gradually getting younger, and as they get older, they have secured a certain support group in terms of self-management. Marketing strategies that stimulate consumers' desires, from advertisements emphasizing medical professionalism, advertisements before and after plastic surgery, to emotional stimulation advertisements, typography advertisements, and outdoor advertisements, are also showing off a great deal of popularity. Korea, which has secured a position as a global plastic surgery powerhouse, is increasing not only among Koreans but also tourists for plastic surgery purposes. When considering the number of plastic surgeries performed by Koreans, even if we apply the method of subtracting the number of visitors for plastic surgery to the number of plastic surgeries performed in Korea, we can guess the increase or decrease in the number.

Looking at the statistics of the International Society of Aesthetic Plastic Surgery (ISAPS) as an example, the total number of plastic surgeries and surgeries performed in Korea in 2010 was 531,425, and in 2014, it was 980,313. According to the Korea Tourism Organization, the number of medical tourists in 2014 is 266,501, so if the purpose of all medical tourism is plastic surgery, it can be expected that the number of cosmetic surgery procedures and surgeries performed only by Koreans is 713,812 .

In 2010 , the number of medical tourists was 81,789 , of which the proportion of cosmetic tourists is unknown, so it is divided into a case where it is assumed that they all did not have plastic surgery and a case where it is assumed that they had plastic surgery, the number of plastic surgeries performed by Koreans in 2010 will be between 449,636 and 531,425.

Based on these results, it can be estimated that the number of plastic surgeries among Koreans increased by about 182,387-264,176 for a total of five years from 2010 to 2014 . There is a reason for trying to figure out the actual plastic surgery population in Korea. Even before the COVID-19 situation, Korea was attracting a lot of tourism for the purpose of plastic surgery, because in fact, the number of plastic surgeries can serve as a basis for understanding one aspect of the plastic surgery powerhouse.

In Korea, under the influence of traditional Confucian ideology, it has been forbidden to artificially transform the body given by parents, but now it is transforming into a kind of aesthetic-oriented era that prioritizes beauty above all else. Even in terms of self-satisfaction or as a purposive method for advancement into society, plastic surgery is breaking away from the conservative perception and forming a positive trajectory in terms of competitiveness. Moreover, the increase in personal income, the one-person media system, the surprising change of video culture by mass media, the priority of appearance, and the expansion of awareness of appearance is competitiveness, etc., helped to secure a public base. 
In modern society, with the development of open online content and mass media, the cycle of trends is fast and diverse, and the desire to show off the beauty of the body is widespread. As the perception of the beauty of the body as one of the realms of a kind of ability or capital increases, the rejection of plastic surgery decreases, and personal beauty is becoming an investment target. According to the logic of being part of human capital, plastic surgery for cosmetic purposes could be expanded. There were many negative views on people who wanted plastic surgery in the past, but recently, plastic surgery is increasing for more practical reasons as a way to increase their value for self-satisfaction, various interviews, and smooth social life.

As revealed in this survey, such a tendency is identified at a high level. As a result of a survey of 271 students at H High School in Gyeonggi-do, 43 responded that they "completely agree" with cosmetic surgery as an individual choice regardless of the surgical site, frequency, or degree. A total of 126 people were positive about one or two places and said that they "generally agree" with cosmetic surgery. While a total of 169 people, or about $62 \%$, expressed their support for cosmetic surgery.

This tendency presupposes the fact that the standardized aesthetic standard is already taken for granted to some extent among young people. It is also possible to approach whether the fact that our society exposes a certain track of aesthetic beauty from adolescence and fails to satisfy it is a form of personal problem. In terms of social competitiveness and equality of opportunity, there is enough room to explore the realistic desires of plastic surgery. This consciousness of the respondent can read the reality of forcing changes to meet aesthetic standards, which are sometimes harsh on individuals in the field of job satisfaction.

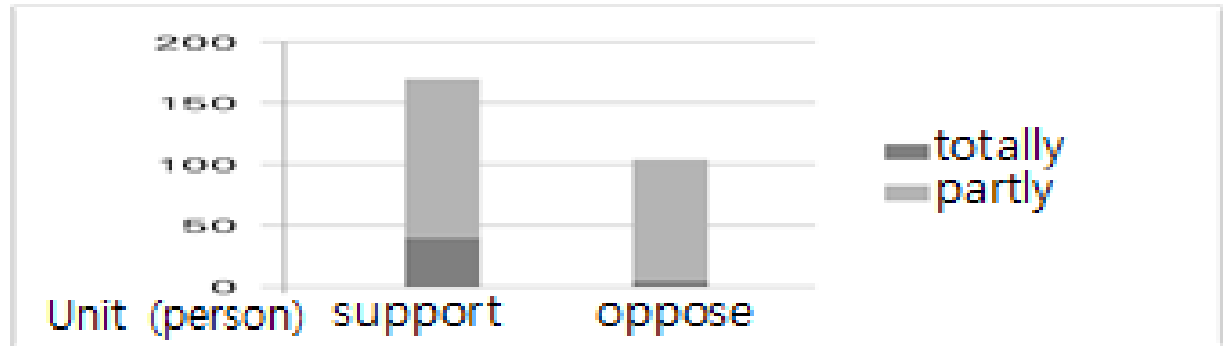

[Fig. 1] Position on Cosmetic Surgery

\section{Early Plastic Surgery and Employment Plastic Surgery}

It is said that youth plastic surgery, which is commonly found in plastic surgery, is often used during vacation after the CSAT. Recently, plastic surgery ads such as "If you bring a test ticket, you will get a special discount" have been criticized for being an irresistible temptation for teenagers and excessive plastic surgery[9]. This type of plastic surgery advertising marketing appears a lot in our society. It is said that excessive plastic surgery for adolescents undergoing growth can lead to physical deformation during their growth period, and that they can be exposed to problems of complications that result in severe growth disorders. Moreover, bone-cutting surgery needs to be decided carefully.

In general, it is easy to judge that bone growth has also been completed if the kidneys no longer grow. In the case of facial skeletons, they grow one to two years later than other bones, so they continue to grow even after their kidneys are fully grown. Therefore, after surgery, problems can arise that appeal to deformities or unnatural appearances of the face[10]. In severe cases, complications such as not opening your mouth properly or not healing your incised face muscles are said to occur. This risk is not to be overlooked. Of course, there is a lot of conflict between consumers and the 
medical community. The problem is that society should fully recognize the problem of risk to teenagers and a forum for public debate should be set up at the social level or at the educational level so that they can have a good understanding of plastic surgery.

In retrospect, there is the urgency to carry out plastic surgery at the risk of surgery and side effects. That is the case for employment after graduation, and in a situation where the employment shortage is prolonged, beautiful appearance is a specification, and it has more power than that for employment. In other words, it is undeniable that appearance plays an important part in the various criteria for evaluating a person. The era has come when people have to prepare for employment plastic surgery to find a job[11]. In 2014, the Presidential Youth Commission's "2030 Policy Participation Group Spec Research Team" announced nine types of specifications that need to be built for employment, adding plastic surgery as a necessary factor for employment.

Figure 2 shows a report on "perception of appearance management and plastic surgery" conducted by Job Korea, a job portal, in September 2018. The results of the survey showed that $34.3 \%$ of the respondents had a positive idea of plastic surgery for employment or relocation. As such, plastic surgery is one of the conditions for employment and is expanding to develop competitiveness.

It is also noted that the proportion of people who actually performed plastic surgery were on the grounds of job placement. Referring to Roh Hyun-hee[12], 22.9\% of plastic surgery reasons in their 20 's answered "for employment" in a survey of 448 people, the second highest after "because they are not confident in their appearance." If you expand your appearance, it is also meaningful that you are not confident in your appearance, but the result is overwhelming compared to those in their 30s $(4.1 \%)$ and 40 s or older $(2.7 \%)$.

According to <SARAMIN>, "Appearance is also an important specification (67.2\%)" in the survey among 1,090 people. People say that the reason for job placement is for confidence from a beautiful appearance. Or, in fierce competition, appearance is the ability to be chosen and will be one that increases one's competitiveness.

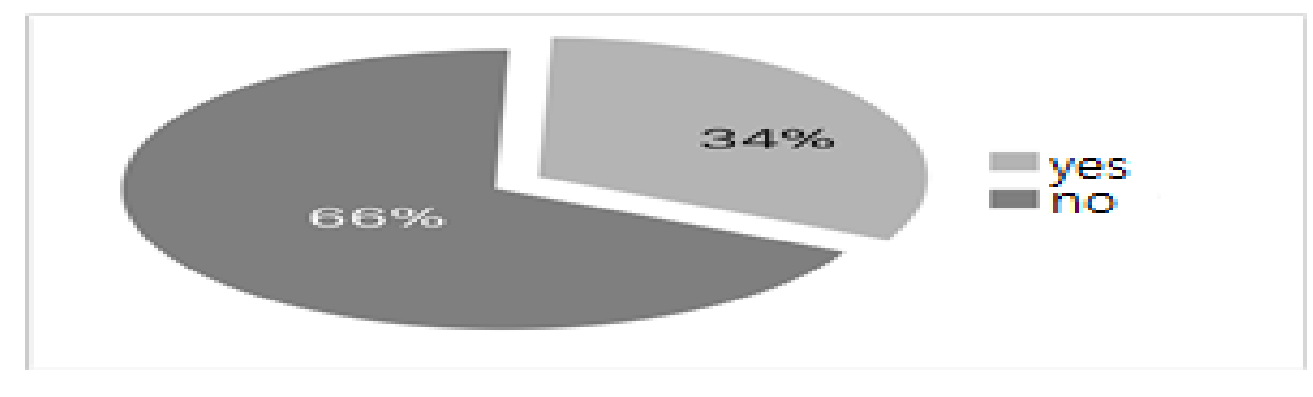

[Fig. 2] Mind to Get Plastic Surgery for Employment and Reemployment

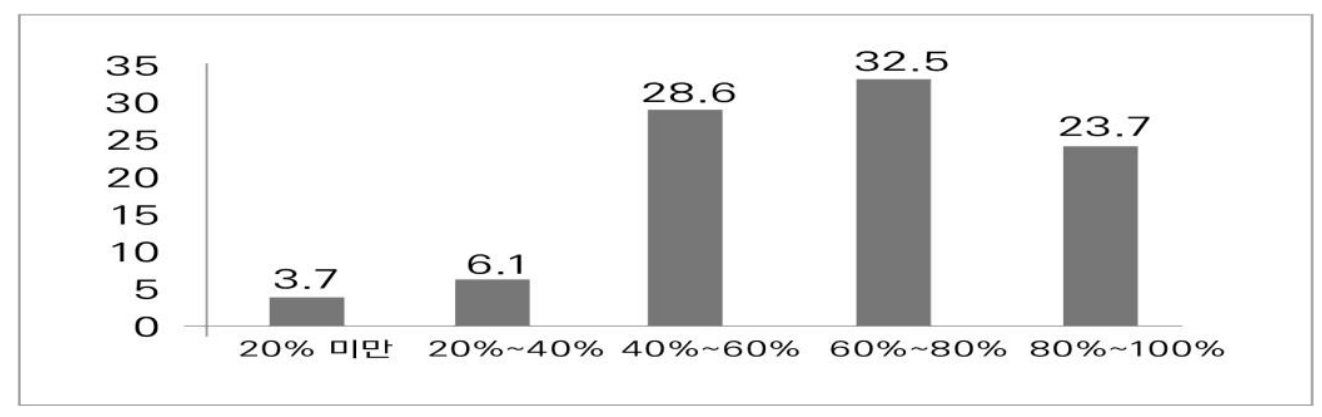

[Fig. 3] Part-time Job and Appearance Recognition Survey 
A survey on 818 college students conducted by ALBACHUNKOOK showed that their appearance has more than half the influence accounting for $56.2 \%$ (499 people) and $84.2 \%$ (688 people). In fact, appearance has more influence than other evaluation factors in the job search process. There will be differences depending on the occupation, but in the case of office workers, it has been confirmed that their appearance has enormous influence in addition to their practical work skills. It is not a desirable direction for appearance to be established as another specification beyond social ability.

\section{Presenting and Analyzing Data}

\subsection{Cosmetic Plastic Surgery and Job Plastic Surgery}

Based on the results of the survey and from prior research, the details of plastic surgery for beauty molding and employment are as follows.

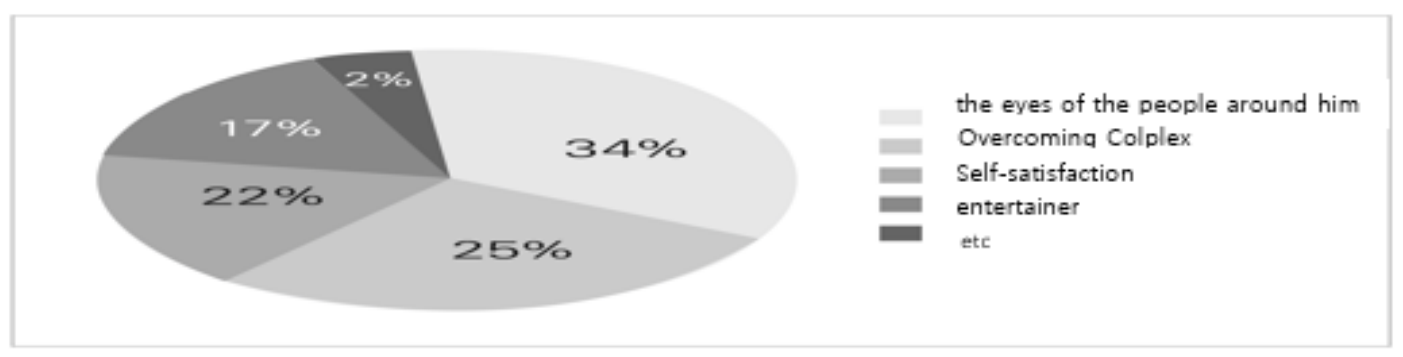

[Fig. 4] Causes of Plastic Surgery in Some Adolescents and Factors that Influence their Choices

Through the figure, we could find common facts in the "the eyes of the people around him", "overcoming complex", and "self-satisfaction" categories that many chose. Many continue to evaluate whether they meet certain 'beautiful standards' of society.

First, "the eyes of the people around him" means the fact that others value themselves for their appearance, and that discrimination, or at least that psychology, is involved.

Second, "overcoming complex" includes recognizing aesthetic standards as "unsuitable" elements of one's appearance, which are so-called "abnormal" or "ugly." That is, it reflects the state of mind that is trying to remove it.

Third, 'self-satisfaction' is similar to 'overcoming complex' but can be seen as a desire to acquire an ideal appearance. It can be seen as a so-called selective aspect of one's appearance that wants improvement in the "not reaching" part of one's aesthetic standards. Eventually, a common sense can be found in the semantics of all the top categories, in that they share a "uniform aesthetic standard."

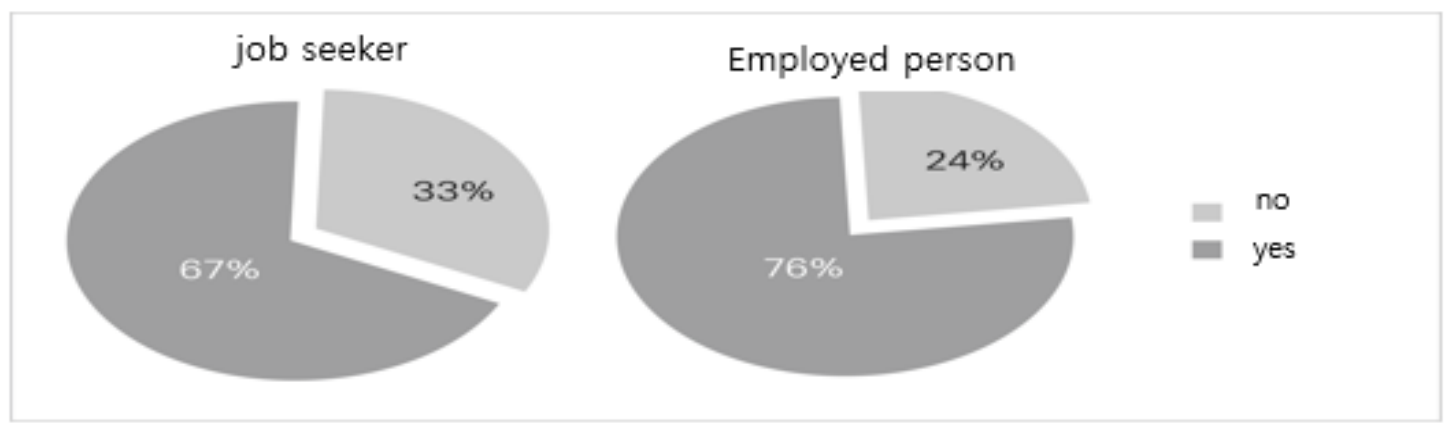

[Fig. 5] The Degree to which one Cares about One's Appearance in Employment or Turnover 
B plastic surgery has conducted a survey of 164 male and female workers and job seekers in their $20 \mathrm{~s}$ and $40 \mathrm{~s}$ on "the extent to which they care about their appearance when they get a job or move jobs." In document screening or interviews, 75.6\% (90 person) respondents said they could be disadvantaged for their appearance, while 66.7\% (30person) said so for job seekers[9].

Based on the above results, it can be seen that employees who actually work was more sensitive to disadvantages caused by their appearance than those who were preparing for employment. This reaction is believed to be due to the fact that the employee has already experienced a direct and indirect impact on social activities by his or her likable appearance, which is formed constantly in social life. Data to understand these results can be identified by reference to previous 2011 surveys. The "motivation to decide to operate" announced by the P plastic surgery and the NHI New Hair Transplant Center for 586 people says $52 \%$ of the respondents decided to operate because of employment or social life. In addition, appearance has a greater impact than anything else for employment and social life, and $96 \%$ of the respondents said so.

However, research that can understand this context can also be found in companies' hiring of personnel. In 2015, a survey of 880 people in charge of human resources at companies conducted by online job portal People showed that $63.8 \%$ of respondents said they would "evaluate the appearance of applicants when hiring." It cannot be overlooked that appearance is reflected in employment quite a bit, as has been discussed in many ways. For that reason, you may think about two things.

Furthermore, $56.9 \%$ of the 880 corporate personnel managers said, "There are applicants who have deducted or eliminated points because of their appearance." Among them, 51\% said they have experience in giving or passing points because of their good looks even if they lack specifications. In the process of finding a job, discriminatory assessment of appearance beyond specification exists in reality. In fact, the reality problem beyond this is limited by individual efforts alone. These experiences of office workers and job seekers act as encouraging cosmetic surgery or job molding at the job site.

\subsection{Early Plastic Surgery and Media Impact}

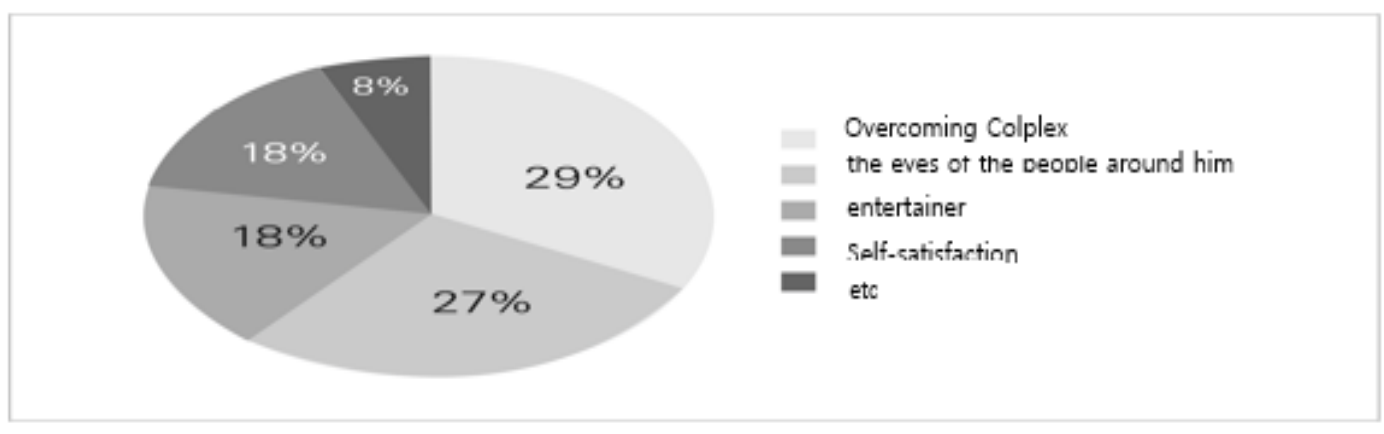

[Fig. 6] Causes of Plastic Surgery in Adolescents and Factors Influencing Choice

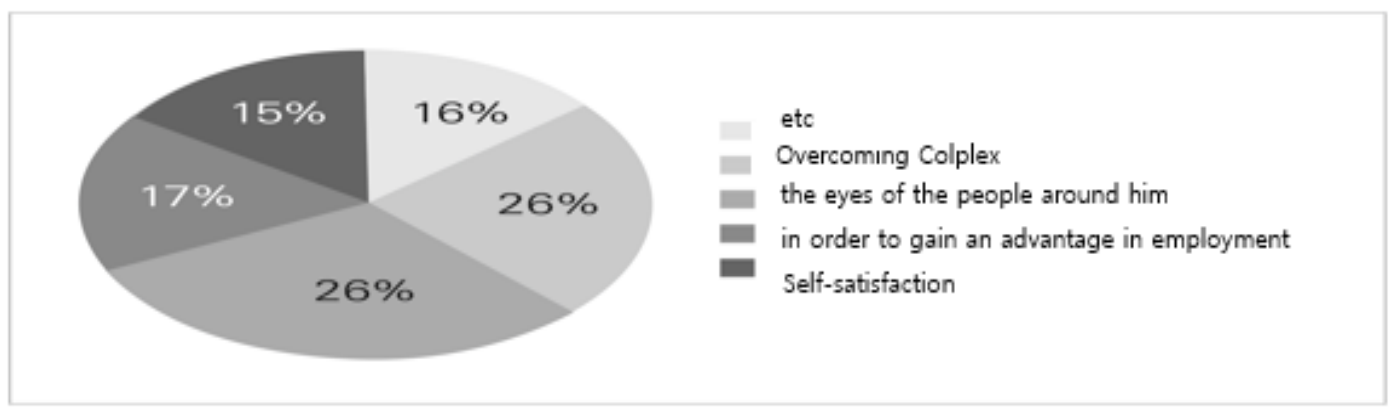

[Fig. 7] Causes of Plastic Surgery and Factors that Influence People's Choice 


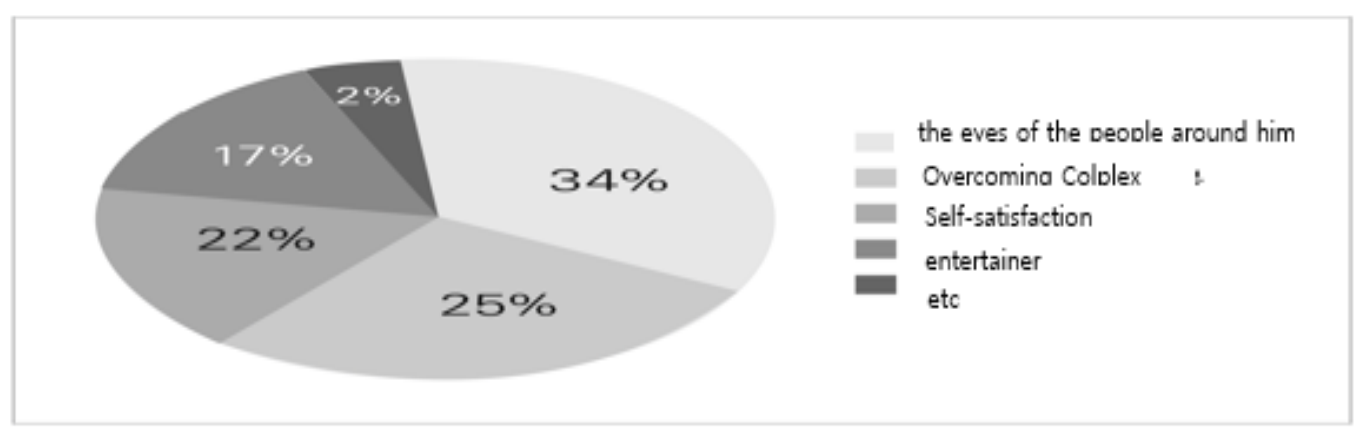

[Fig. 8] Causes of Plastic Surgery in Adolescents and Factors Influencing Choice

Among the reasons why adults choose plastic surgery, celebrities show low figures of $5.13 \%$ and 9.6\%, respectively, when selecting maximum (single-response) and all (multiple-response) factors. However, in the case of teenagers, $17 \%$ and $18 \%$ of the survey asked why the item "celebrity" was plastic surgery, which was relatively high. This means that as a public figure, celebrities have a higher impact on their appearance than adults because of their large exposure and ripple effects to young people who grow and absorb quickly.

In addition, with the advent of various audition programs, plastic surgery is recognized by teenagers as a competitive power to enter society or to get what they want. It is more than imitation psychology for teenagers who are now interested in and followed not only by their appearance but also by their financial sense of envy and deprivation[14]. Moreover, the appearance of celebrities who boast about their changed appearance through plastic surgery is no longer unfamiliar in our society because it is a common conversation through the media.

It is certainly not easy for young people to be free from plastic surgery as their friends and peers have beautiful looks through plastic surgery and continuously receive information related to it through various media. Adolescents participate and communicate through mass media and SNS compared to other age groups, so they are exposed to a considerable amount of time and gain a lot of information and knowledge from them[15].

Adolescence is a state in which self-identification is not fully established, and aesthetic values are often dependent on external factors. However, the media frequently appears in various programs by putting people who meet certain standards of appearance, making them popular and emphasizing their appearance. It also gives laughter through people who look relatively lacking in aesthetic standards. This is no exception not only for entertainment but also for programs centered around couples. Of course, we do not know the specificity of the media that needs to consider ratings. As a result, this appearance of the media provides awareness that adolescents are unknowingly exposed and stereotyped to uniform aesthetic standards[11]. Public and public-interest-based media should, among other things, expand the provision of unbiased perception in all areas of attractiveness and body image resulting from differences in physical conditions.

As the appearance-oriented figure exposed through mass media is formulated as a pursuit of happiness and success, skinny physical conditions are emphasized as ideal. These various advertising marketing of mass media stimulate individuals' values and desire to change their bodies[8][16]. Even teenagers who have no experience of direct discrimination due to their appearance stand out because they cannot overlook the influence of the media that access information other than school or home.

As a result, the media has exercised the influence to expose and disseminate certain aesthetic standards that prevails in society or are constantly required in society. Media outlets in the Ubiquitous era are moving uniform standards to the world faster and stronger. Sometimes the public admires and is enthusiastic about people who meet these aesthetic standards. The public may show excessive 
interest in appearance to ride on social standards on their own, or not to be left out of this trend. However, the aesthetic standards that the media shows may be out of touch with reality. The reality is that there are elements that are hard to achieve for the public.

\section{Conclusion}

Today's society exposes certain aesthetic standards, whether consciously or unconsciously, and requires members of the society to do so. In a flood of aesthetic standards and beauty that continues without hesitation, people come to regard these aesthetic standards as if they were official, and they are trying to fit themselves into the standards. The process naturally encouraged plastic surgery, and the perception of lookism that prevailed in society became a social pathology phenomenon beyond an individual problem.

In fact, each person's appearance is diverse and individual is endowed with individual beauty. However, modern society does not have a life of its own, and is immersed in the aesthetic standards provided by society which shows itself to be standardized.

Rather than forcing the aesthetic standards exposed by society, there is an urgent need to restore self-esteem that can increase competitiveness and an attitude to take pride in one's appearance. In addition, it is necessary to supplement the regulatory measures that will reduce the harmful effects of such social perception and realistically check the irrational structure.

First, there is an urgent need to regulate indiscriminate and sometimes overly stimulating public transportation and Internet plastic surgery advertisements. Recently, plastic surgery advertisements are not limited to banner advertisements appearing on portal sites or articles, but also advertisements in the form of reviews that appear to have been written by other consumers in similar situations are increasing.

Natural advertisements in the form of word of mouth, a type of "viral marketing," can give people trust, clouding or shaking consumer judgment. Advertisements through mobile web and applications are also on the rise, making this problem even more diverse. However, the subject of 'Medical PreAdvertising Review' enforced by the current Medical Act does not include advertisements of a late nature, mobile web or mobile application advertisements. In reality, media that have a large impact on consumers and are highly accessible are not properly regulated. Even plastic surgery advertisements on the Internet or mobile applications, which are easier to access than public transportation, need to be improved urgently with tight management, supervision, and regulation.

Second, the programming or introduction of provocative plastic surgery-related TV programs centered on viewership also requires a significant amount of regulation. At least, it is necessary to refrain from organizing programs that correspond to the youth age. Competitively encouraging plastic surgery through TV and the text selected for broadcasting is more than a fresh shock, the intensity of the stimulus is deepening. These plastic surgery-related programs are a one-way process, and by showing viewers only the positive aspects of plastic surgery, they provoke a desire for plastic surgery. In the end, such exposure increases people's unnecessary plastic surgery and surgery, and even TV plastic surgery programs are stolen as advertisements.

Third, it is the regulation of reckless plastic surgery behavior of adolescents under the age of 19 that needs supervision. Youth are in a period of physical, mental, and growth growing day by day. Plastic surgery can affect the growth of bone or cartilage, and there is a huge risk that the body can be deformed during the growth process. The medical community also recommends that plastic surgery be performed after growth is completed.

Fourth, it is necessary to apply multi-faceted regulations and changed manuals to areas that can be defined by appearance evaluation when hiring. Because appearance does not necessarily have to take precedence, the interview content and evaluation method are substituted for different interview 
contents and evaluation methods according to the work area and specificity, and the appearanceoriented interview items are excluded or assigned as reference.

Fifth, it is necessary to introduce full-scale blind interviews to corporations and small and mediumsized enterprises as well as public institutions, to exclude resume photos, to apply various evaluation items in addition to external evaluation, and to operate and expand mock interview training programs. The details applicable here are as follows.

First, various companies and institutions are operating a training course to become an official member, equipped with realism, practicality, and practical skills beyond the internship system. Through this, companies or institutions can recruit the necessary human resources and technical manpower and pursue a direction to increase social interest and competitiveness.

In terms of diversification of evaluation, evaluation of problem-solving ability and creativity related to work characteristics, self-development performance, responsibility and collaboration ability, futureoriented possibility, service area, expansion of experience and results, awareness of social order, and sense of social justice or ethics should be considered as hiring evaluation items and methods to suit the characteristics of the job. In addition, it is necessary to consider the introduction of qualitative and quantitative evaluation and the balanced application of non-examination curriculum as evaluation items in the case of qualifications related to work.

Finally, it is necessary to consider the case of re-education of interviewers. In fact, as a result of a 2012 job portal survey of 776 Corporate Human Resources Managers by SARAMIN, 59.6\% of these companies said that they had experienced rejecting applicants who did not attach a photo on their job application form. As a result of the 2015 Seoul YMCA's survey, 3 out of the top 30 companies asked job seekers to write down their physical details, such as height and weight.

In a society where appearance management is regarded as self-management, evaluation items that are not related to work even in employment are determining whether or not they are successful. We need to recognize that appearance is not a determining factor in life. Of course, a change in social awareness must be a prerequisite, but the start of such a change will also be a workaround if the change of individual consciousness is not preceded.

As mentioned, it is definitely necessary to have a competitive appearance in order to enter society. The important point is that various social efforts at the individual level towards the goal should not be buried due to physical appearance. This suggestion is meaningful even to find the attractiveness, temperament, character, or the possibility and ability that come from differences.

\section{References}

[1] Ki-bum Kim, Yang-ha Kim, Cha Young-ran, A Narrativitized and Discursive Maum of Socialized Beauty and Body in Korea, Korean Women's Psychological Society, (2007), Vol.12 No.3, pp.337-393.

[2] Park bock soon, Kim Joo Hyun, Structural Equation Modeling Intention of Revision Facial Cosmetic Surgery in Female College Students, Journal of the Korean Society of Industrial-Academic Technology, (2015), Vol.16, No.12, pp.8707-8718.

[3] Hyun-jin Lee, Oh-Ryun Kwon, Dong-IL Han, Study on the body appearing in the interdisciplinary researches as the viewpoint of physical education, Journal of the Korean Sports Philosophy, (2016), Vol.24, No.1, pp.183-197.

[4] Chen Song chao, B. Sujatha, G. Karuna, Performance measures for Image Fusion based on Top-hat transform and Wavelet transform, International Journal of Smart Business and Technology, (2014), Vol.2, No.2, pp.1-10.

[5] Hyun-ah Kim, Seung-ah Ryu, Choi Soo-kyung, A Study on the Relationship between Appearance Management Behavior and Career Determination Self-Efficacy of Female College Students in Changwon Province, The Journal of the Korean Design and Culture, (2019), Vol.25, No.3, pp.87-99. 
[6] Ae-hwa Hwang, Ji-woong Kang, Samsung Han, Analysis of University Students' Experience on Cosmetic Surgery and Skin Care, The Journal of the Korean Beauty, (2017), Vol.13, No.2, pp.125-132.

[7] Che-Rin Park, Chae-Jeong Han, Effect of Beauty Lifestyle Behaviors on the Pursuit of Beauty Values and Cosmetics Purchasing Behaviors, Convergence Information Paper, (2021), Vol.11, No.1, pp.261-267.

[8] Min-ji Kang, Kyung-hyun Lee, Study on the consumer behavior in different types of outdoor advertising of cosmetic surgery centers-Special focus on outdoor advertisements in subway stations of Gangnam region, Korea Design Forum, (2014), No.44, pp.315-325.

[9] Won-ik Cho, Employees are more sensitive to their looks when looking for jobs than job seekers, Sports World, September, (2014), http://m.sportsworldi.com/view/20141105004854

[10] https://news.v.daum.net/v/20150112150307395, January 12, (2015)

[11] https://www.anewsa.com/news_print_paper.php?number=944410, December 23, (2015)

[12] Hyun-hee Noh, A Study on the Recognition and Actual Condition of Cosmetic Plastic Surgery in Adult Women's University, Sookmyung Women's University, Master's Thesis, (2014)

[14] Chul Cho, Jin-seop Roh, Hye-mi Choi, Why do you keep putting a knife on a bright face, Sisa Journal, July, (2013)

[15] Eun-kyung Na, Joo-hyun Hong, Social Psychology of Watching Plastic and Beauty Reality Programs and Using Social Media: Effects of High School Girl's Body Image and Censorship, Social Comparison and Self-Satisfaction, (2018), Vol.26, No.1, pp.71-96.

[16] Hyun-sook Kim, The Effect of Perception of the Justification of Appearance Efforts and Plastic Benefits on Plastic Behavior: Focusing on Double Standards for Himself and Others, Korean Design and Culture Journal, (2014), Vol.20, No.4, pp.153-163. 